\title{
Modeling and Parameter Estimation of Particle Swarm Optimization Algorithm for Smart Power Grid
}

\author{
He Tao ${ }^{1}$, Liang Zhidong ${ }^{2, *}$, Ye Xinquan ${ }^{3}$, Sun Shufeng ${ }^{3}$ and Pang Jihong ${ }^{3}$ \\ ${ }^{1}$ Oujiang College of Wenzhou University, Wenzhou325035, P.R.CHINA; \\ ${ }^{2}$ School of management, Shangdong Youth University of Political Science, Jinan \\ 250103, P. R. China \\ 3. College of Mechanical \& Electrical Engineering, Wenzhou University, \\ Wenzhou325035, P.R.CHINA \\ 1254166017@qq.com
}

\begin{abstract}
This paper analysis the electric power communication network structure and performance requirements. To investigate the power communication backbone network and network power substation general way, and in electric power communication network delay, error rate, network bandwidth demand were analyzed. The for prevalent in electric power communication network is not a fully meshed network, explore artificially cobweb logic change principle and system method, artificial transform sheet mesh to the standard web, and making use of the cobweb, based on the characteristics of network fast routing. In order to better in the smart grid communication network using artificially cobweb routing algorithm, for the smart grid in the presence of irregular mesh network, this paper presents a kind of the mesh network into logical artificially cobweb network general standardization method. The principle of combination of label switching, this paper presents artificially cobweb source routing algorithm, expounds the artificially cobweb routing algorithm of label switching and label update mechanism, and artificially cobweb routing algorithm balancing routing method and routing method is described in detail.
\end{abstract}

Keywords: Nonstationary signal; Stable distribution; Time-frequency autoregressive; Time-frequency spectrum

\section{Introduction}

Particle swarm optimization (PSO) algorithm is the middle of 90's, Dr. Eberhart and Dr. Kennedy [1-6], a new swarm intelligence [7] co inventor of the computing technology, which originated from the research of bird and fish behavior. The basic idea of particle swarm optimization algorithm is through collaboration and information sharing between individuals in groups to find the optimal solution. PSO algorithm is simple, easy to implement, fast search speed, search range, compared with other algorithms, it has outstanding advantages. It is a random search algorithm parallel global in nature [8-11]. For the electric power communication network management complexity will play a positive role, this paper attempts to apply the PSO algorithm to the power communication network management to find the best repair path and the optimization of route selection, the selection of the best path routing to provide quality of service and better network load balance. It's very important to promote the research and development of network management of electric power communication network [12].

Liang Zhidong is the corresponding author. 
Optimization problem exists in all aspects of human life, since people are looking for all types of appropriate optimization algorithm to solve a variety of optimization problems. With the widening of the various application fields, some optimization problems that arise has become more and more complex, the corresponding optimization methods for these optimization problems also from some of the traditional optimization method to intelligent optimization algorithm and the particle swarm optimization algorithm (PSO) as a branch of swarm intelligence, PSO algorithm origin in scientific research of birds foraging behavior of this group activities study. Birds foraging in the flight process will collective collaboration is guaranteed to find food, is also based on this point, the PSO algorithm is by individuals in the current population of mutual cooperation, the formation of an information sharing mechanism, the exchange of information, so as to ensure the whole particle swarm can find the global optimal solution. Because of the ideological structure of PSO algorithm is simple and easy to implement, run a few parameters and the convergence speed is fast advantages, more and more people have paid close attention to, has been widely used in scientific research and engineering application [13].

The concept is simple, easy to implement, and the need to adjust the parameters of the advantages of less, caused the people's extensive concern, and quickly became a new hotspot. Because of its shorter development time, the research is still in the initial stage, there are still many problems to be studied. The on the basis of comprehensive analysis of the standard PSO algorithm, in order to improve the optimization performance of the algorithm as the ultimate goal, the theory of the algorithm made in-depth study, and puts forward the corresponding improvement of the PSO algorithm, and applied in practice.

\section{The Research Status of Smart Power Grid Network}

Although the earth shaking changes have taken place in the past decades, but traditional grid and not to follow the time the step. Smart grid integration of new control technology, information technology, power technology and management technology, with high efficiency, safe and reliable flexible characteristics become in today's world, the development of power system and the change trend.

In Europe, since the European Union countries attaches great importance to environmental protection, smart grid research more focused on renewable energy access and distributed generation. In 2005 [14], the European Commission set up a "smart grid European technology forum", the purpose is to transform into power grid users and operation of the interactive service network, improve the transmission and distribution system of efficiency, safety and reliability. In 2006 [15], the EU has issued the European sustainable, competitive and secure energy strategy "report report emphasizes the Europe have entered the new energy era, and the development of smart grid is that power could be the key to the quality and development. Mainly from the network assets, grid operation, the demand side and metering, power generation and electricity energy storage in four aspects of smart grid research.

Although the technology of smart grid in China in recent years has been rapid development, but smart grid communication backbone network is not strong, smart grid communication technology still faces many problems, it is difficult to completely satisfy the new requirements for scheduling data communication network, the second plane construction. At the same time distribution, power link level of communication with relative transmission gap larger, at all levels of the communication network resources integration and make full use of needs to be further strengthened. 


\section{The Basic Theory of Particle Swarm Optimization Algorithm}

\section{1 $\alpha$ Stable Distribution}

$\alpha$ stable distribution is a kind of generalized Gaussian distribution, the process is not limited in variance and their probability density function has a serious tail, its characteristic function can be described as[6-8]

$$
\varphi(t)=\exp \left\{j \mu t-\gamma|t|^{\alpha}[1+j \beta \operatorname{sign}(t) \omega(\tau, \alpha)]\right\}
$$

$$
\text { Where, } \quad \omega(\tau, \alpha)=\left\{\begin{array}{ll}
\tan (\alpha \pi / 2) & \text { if } \alpha \neq 1 \\
(2 / \pi) \log |\tau| & \text { if } \alpha=1
\end{array} \quad \text { sign }(t)=\left\{\begin{array}{cc}
1 & t>0 \\
0 & t=0 \\
-1 & t<0
\end{array}, \quad \alpha \quad\right. \text { is the }\right.
$$

characteristic index, when $0<\alpha<2,(1)$ is lower order stable distribution, when $\alpha=2$, it is Gaussian distribution. $\beta$ is the symmetry coefficient, $\gamma$ is the dispersion coefficient, $\mu$ is the location parameter. When $\beta=0, \mu=0, \gamma=1$, it is called the standard symmetric $\alpha$ stable distribution ( $S \alpha S$ ). When $\alpha=1.0,1.5$ and 2.0, the timedomain waveform of $s \alpha S$ stable distribution in Figure 1.
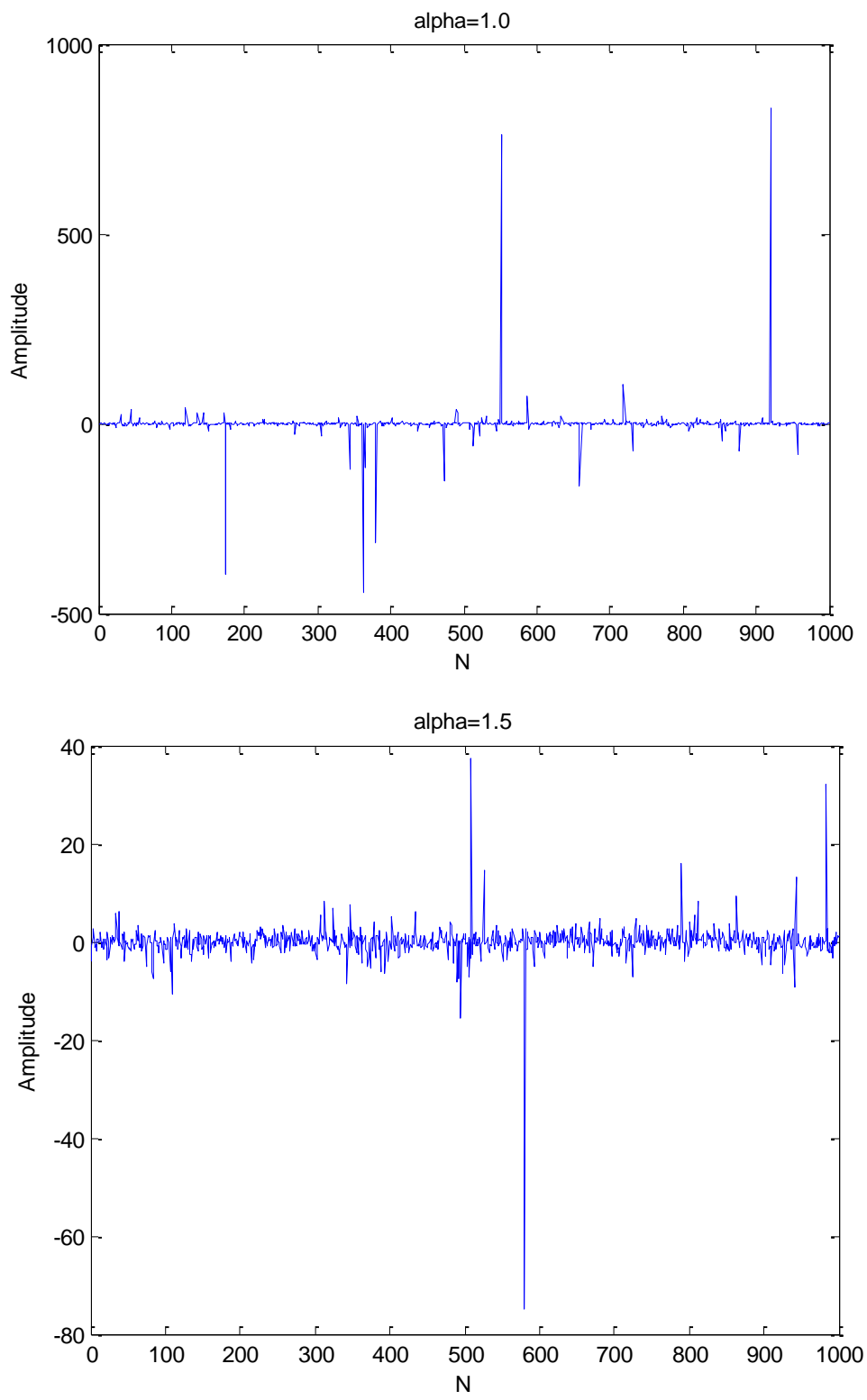


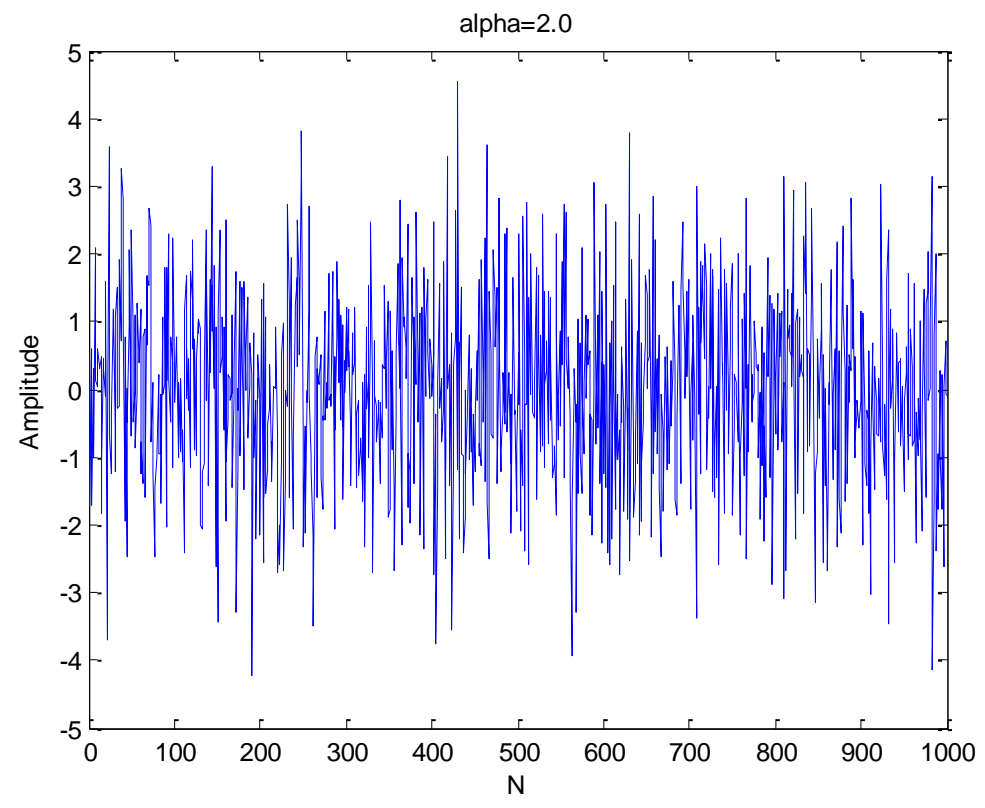

Figure 1. Time-Domain Waveform of Stable Distribution Under $\alpha=1.0,1.5$ and 2.0

\subsection{PSO Algorithm Flow}

A cluster is usually defined as a collection of a group of autonomous bodies, through their mutual direct or indirect communication.

The steps of the algorithm are described below:

(1) The initialization of particle swarm optimization, namely random set each particle initial position and initial speed of $\mathrm{V} \mathrm{x}$;

(2) The calculation of the fitness value of each particle;

(3) The comparison of each particle, its fitness value and the best position of pbest has the fitness value; if better, update pbest;

(4) The best position of the gbest experience and the degree of population fitness value; if better, update gbest;

(5) Based on the particle position and velocity updating formula to adjust the position and velocity;

(6) If you reach the end of the conditions (good enough or the location of the maximum number of iterations), end; otherwise, go to step 2.

According to the basic steps of PSO algorithm is difficult to get the flow diagram, as shown in Figure 2. 


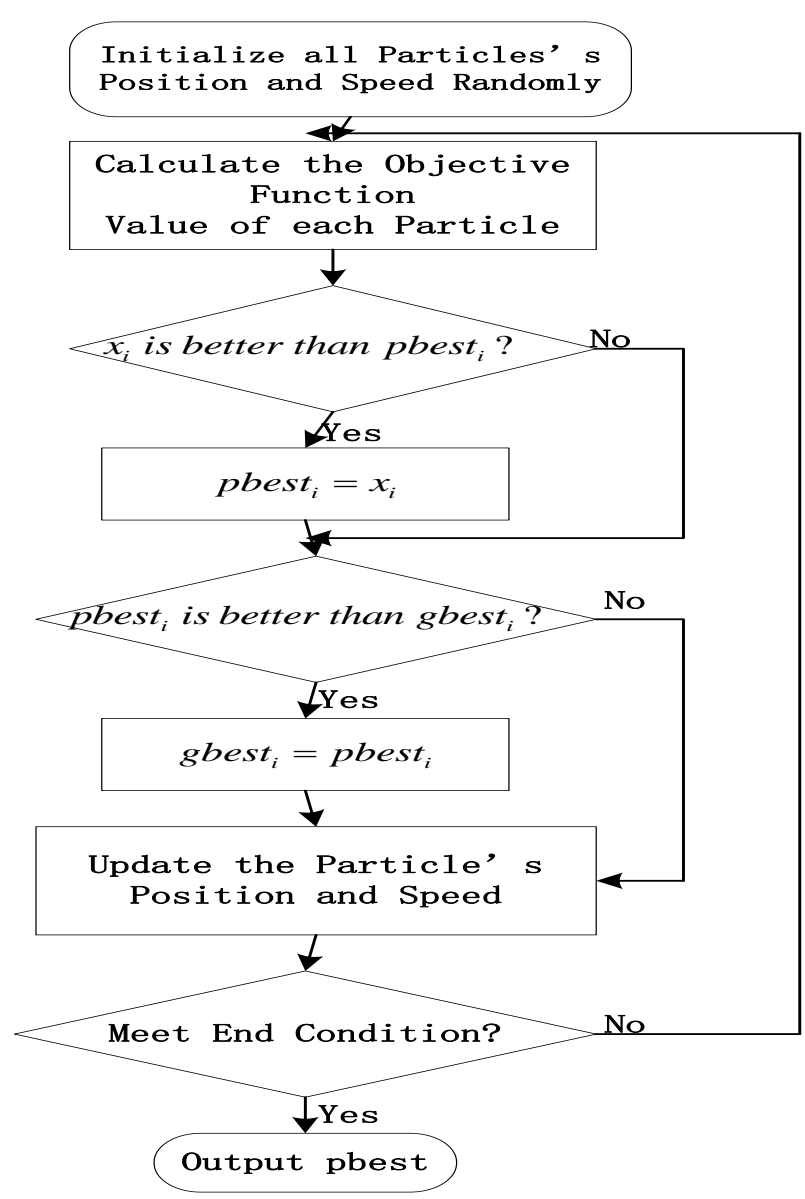

Figure 2. The PSO Algorithm Flow Chart

The choice of parent and not based on fitness, the fitness selection is likely to fall into the local optimal risk with a large number of local extreme value problem solution based on the. The probability of $\mathrm{P}(0,1)$ is a random number between (experience value is about 0.2 ). This algorithm is called the breed type of particle swarm optimization algorithm. Theoretically speaking, BPSO can better search the space between particles, two in different sub optimal peak particle after breeding, can escape from the local optimal. For unimodal functions, BPSO is slightly faster convergence speed, but not as good as the basic PSO and GA solutions, and for a large number of local extreme values of BPSO function, not only speed up the convergence, but also found equally good or better solution.

\section{The Experiment and Analysis}

\subsection{The Fixed Weight and Time-Varying Weights Selection}

The parameters in the algorithm is the key to affect the algorithm performance and efficiency, how to determine the optimal parameters of the best algorithm performance itself is an extremely complex optimization problems. The formula in the inertia weight is an important parameter in the standard version of the PSO algorithm, the implementation effect depends largely on the inertia weight is selected, it is used to control the exploitation and exploration ability. The inertia weight determines the size of the current speed inherited many particles. Larger inertia weight will make the particle has greater speed, and strong ability to explore; inertia weight is smaller the particle has a strong 
development ability. Therefore, it determines the searching capability of the algorithm and implementation of efficiency to a great extent.

The inertia weight is an important parameter in the PSO algorithm, the choice is mainly divided into two categories: fixed weights and variable weights. Fixed weight selection is to select a constant as the weight value, unchanged in the process of optimization, the particle has the exploration and exploitation ability of the same. But when the variable weight is selected for a certain range, with the exploration and exploitation ability in different period in the iterative process of optimization, with a decreasing rate decreases linearly, so the time-varying weight selection range and rate of decline of choice.

The population size of 40 , the maximum number of iterations per run 2000 . PSO optimization process the experimental results were shown in Table 1.

Table 1. PSO Performance under the Weight of the Fixed and Time-Varying

\begin{tabular}{|c|c|c|c|c|c|c|}
\hline parameter & index & function 1 & $\begin{array}{l}\text { function } \\
2\end{array}$ & $\begin{array}{l}\text { function } \\
3\end{array}$ & $\begin{array}{l}\text { function } \\
4\end{array}$ & function 5 \\
\hline \multirow[b]{2}{*}{1} & $\begin{array}{l}\text { excellent } \\
\text { rate }\end{array}$ & 1 & 0.95 & 0.9 & 0.8 & 0.75 \\
\hline & $\begin{array}{l}\text { average } \\
\text { iteration } \\
\text { number }\end{array}$ & 350.2 & 547.4 & 130.2 & 300.1 & 480 \\
\hline \multirow[b]{2}{*}{2} & $\begin{array}{l}\text { excellent } \\
\text { rate }\end{array}$ & 1 & 0.9 & 0.95 & 1 & 0.8 \\
\hline & $\begin{array}{l}\text { average } \\
\text { iteration } \\
\text { number }\end{array}$ & 380 & 763.7 & 240.3 & 354.2 & 410.2 \\
\hline \multirow[b]{2}{*}{3} & $\begin{array}{l}\text { excellent } \\
\text { rate }\end{array}$ & 1 & 0.70 & 0.95 & 1 & 0.85 \\
\hline & $\begin{array}{l}\text { average } \\
\text { iteration } \\
\text { number }\end{array}$ & 1001.3 & 1120.5 & 998 & 1203 & 880.6 \\
\hline \multirow[b]{2}{*}{4} & $\begin{array}{l}\text { excellent } \\
\text { rate }\end{array}$ & 1 & 0.85 & 0.95 & 1 & 0.85 \\
\hline & $\begin{array}{l}\text { average } \\
\text { iteration } \\
\text { number }\end{array}$ & 475.2 & 650.5 & 432.1 & 694 & 443.2 \\
\hline
\end{tabular}

\subsection{Analysis of Double Star Network Simulation}

Double star network in the normal and sudden traffic, the network's status is similar with the star network. In the simulation, we set up in the 500ms time, the link between node1 and hub1 interrupt.

In Figure 3, the blue curve and red curves respectively represent the flow of node1hub1 node1-hub2, in Figure 4, blue curve and red curves represent star network with single artificially cobweb delay.

As shown in Figure 4 in less than 500s. During normal operation of the network, star shaped network on the main path of flow and nodes contract rate has been, for 8000bps. At this point the data packet without backup path forwarding, traffic on the backup path is 0 . At this time network sales ete delay and monolayer artificially cobweb model, were maintained at around $64 \mathrm{~ms}$. At this time, the star network communication performance and artificially cobweb model similar. 


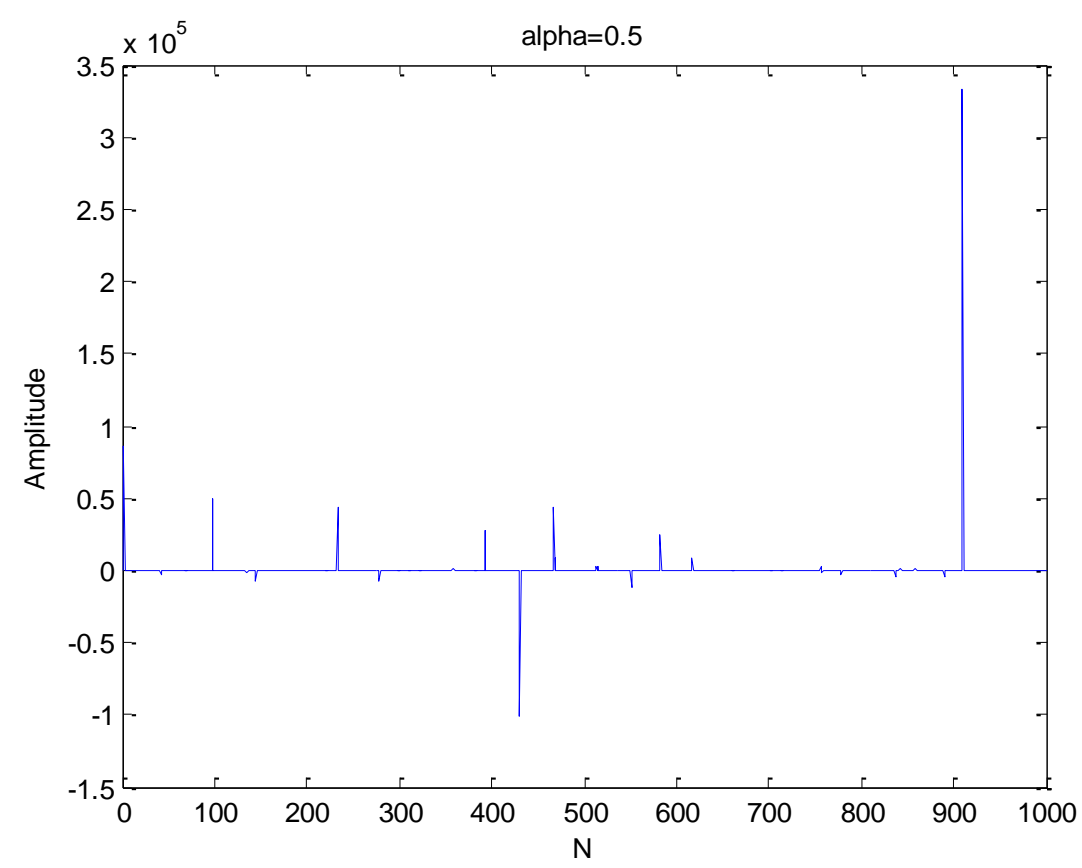

Figure 3. The Blue Curve Represent the Flow of Node1-Hub1 Node1-Hub2

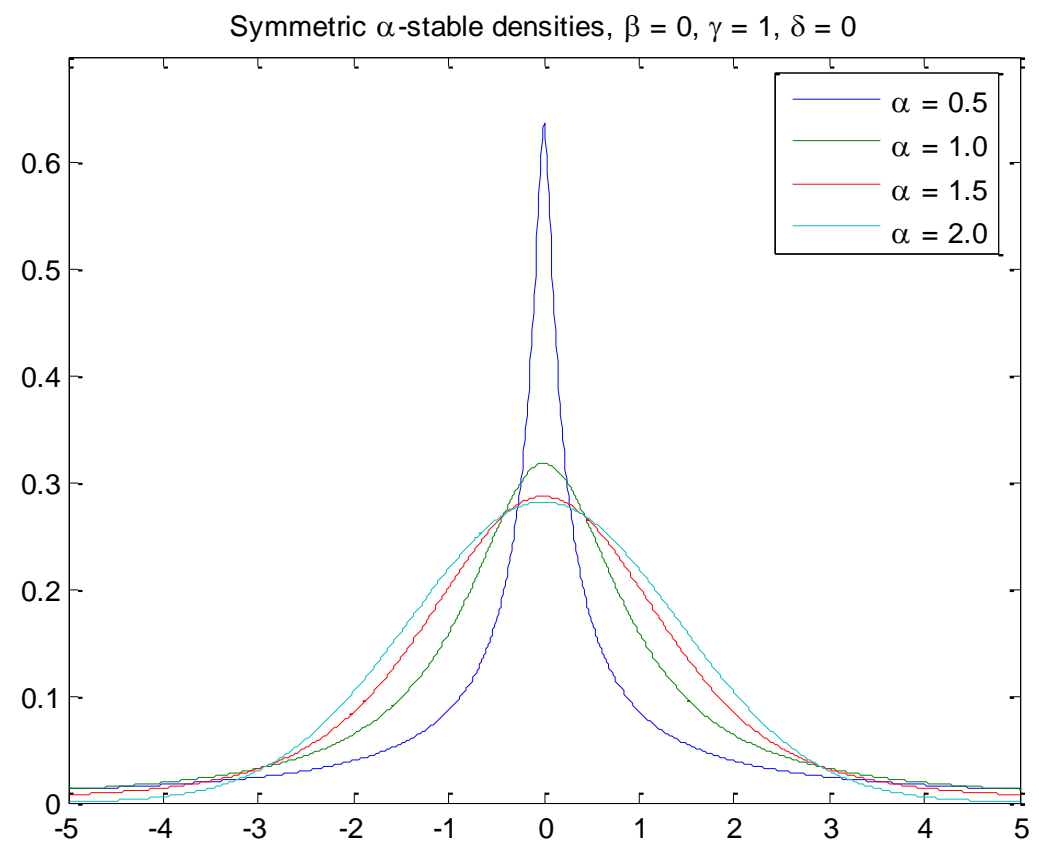

Figure 4. Blue Curve and Red Curves Represent Star Network with Single Artificially Cobweb

Double star shaped network for single path resistance path outage performance than artificially cobweb model good, but in the binary shape model, if the primary path and the backup path and the outage, the network will appear isolated nodes, and in monolayer artificially cobweb, if with peripheral nodes have two link break, the network is still reliable, peripheral node can still through the three link and network to establish a connection. 


\section{Conclusion}

Smart grid mesh network network standardization method and routing algorithm is the main research of this paper, the capacity and reliability of network communication link is the premise of the analysis of the network is consistent, although the results have a certain theoretical significance, but according to the actual characteristics of the smart grid mesh network for the further theoretical analysis is necessary. In artificially cobweb routing algorithms, this paper does not have in-depth study on the traffic engineering, traffic engineering is load balancing, reliable communication is an important method in the network, network is directly related to the overall performance, worthy of more in-depth study.

\section{Acknowledgments}

This work was supported by the National Natural Science Foundation, China (No.11372229, No.71271156, No. 71301120), the Provincial Natural Science Foundation, Zhejiang, China (No.LY13G010002), Science Technology Department of Zhejiang Province (No.2012C14026, No.2012C21050), Education Department of Zhejiang Province (No.Z201120727, No.Y201224800).

\section{References}

[1] X. Liang, C. Tianding, R. Zhiguo, W. Di, "Resource management for uplink OFDM wireless communication system [C]", Global Mobile Congress, (2007), pp. 284-288.

[2] M. Khodier, G. Saleh, "Beamforming and Power control for interference reduction in wireless communications using particle swarm optimization[J]", International Journal of Electronics and Communications, vol. 64, no. 6, (2010), pp.489-502.

[3] A. Radonjie, V. Vujicie, "Integer SEC-DED codes for low power communications [J]", Information Processing Letters ,vol. 110, no. 12-13, (2010), pp. 518-520.

[4] S.wan Kim, J. Park, S. Han, H. Kim, "Development of extended speech act coding scheme to observe communication characteristics of human operators of nuclear Power Plants under abnormal conditions [J]", Journal of Loss Prevention in the ProcessIndustries , vol. 23, no. 4, (2010), pp. 539-548.

[5] A. Vacearo, D. Villacei, "Performance analysis of low earth orbit satellites for Power system communication [J]", Electric Power Systems Researeh , vol. 73, no. 3, pp. 287-294.

[6] Y-j Chung, C-h Paik, H-g Kim, "Sub gradient approach for resource management in multi-user OFDM systems[C]", First International Conference on Communications and Elcetronies, (2007), 5

[7] G. Bader, F. Ben Slimane,S., J. pijoan, "Adaptive resource management for a MC-CDMA system with mixed QoS classes using a cross layer strategy[C]", IEEEVehicularTechnologyConference, (2007), pp. 3036-3040.

[8] C F Juang, "A hybrid of genetic algorithm and particle swarm optimization for recurrent network design[J]", Systems, Man, and Cybernetics, Part B: Cybernetics, IEEE Transactions on, vol. 34, no. (2), (2004), pp. 997-1006.

[9] M. Jachan, .G Matz and FHlawatsch.TFARMA . MODELS: ORDER ESTIMATION AND STABILIZATION [J] . IEEE ICASSP-2005, vol. IV, (2005), pp. 301-304.

[10] M. Jachan, G. Matz and F Hlawatsch, Time-Frequency Autoregressive Random Processes: Modeling and Fast Parameter Estimation[J], IEEE ICASSP-2003,(2003), pp. 125-128.

[11] M. Jachan, G. Matz and F Hlawatsch. Least-squares and Maximum-likelihood TFAR Parameter estimation for nonstarionary processes[J], IEEE ICASSP-2006, vol(III), (2006), pp. 492-495.

[12] M. Jachan, G. Matz and F Hlawatsch. Vector Time-Frequency AR Models for Nonstationary Multivariate Random Processes[J], IEEE TRANSACTIONS ON SIGNAL PROCESSING, vol. 57, no. 12, (2009), pp. 4646-4658.

[13] X. Ma ,C L Nikias, "Parameter estimation and blind channel identification in impulsive signal environments [J]", IEEE Transactions on Signal Processing, vol. 43, no. 12, (1995), pp. 2884 - 2897.

[14] M.shao.C.L.Nikias, "singnal processing with fractional lower order moments instable processed and their applications [J]", proceedings of the IEEE, vol. 81, no. 7, (1993), pp. 986-1010. 


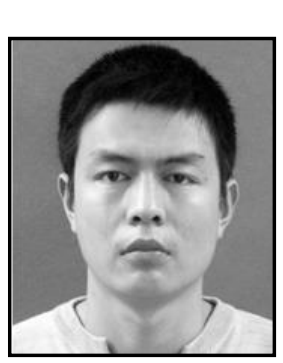

\section{Authors}

He Tao works currently as a Lecturer of Oujiang School of WenZhou University. He received the ME degree in mechanical engineering from WuHan University of Technology, China, in 2008. His major interest is the work of intelligent detection and control, the complex system optimization.

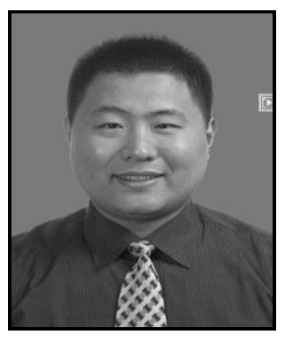

Liang Zhidong works currently as a Lecturer of Management School in Shandong Youth University of Political Science. He has achieved the $\mathrm{PhD}$ in management from THE Hong Kong Polytechnic University. in 2014. His major interest is the work of regional public management, crisis management and management information system.

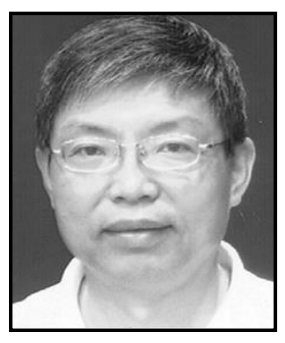

Ye Xianquan works currently as Professor in College of Mechanical and Electrical Engineering, Wenzhou University. He has achieved the BE degree in mechanical engineering from TianJin University of Technology, in 1982. His major interest is the work of High/Low voltage electrical equipment system, the complex system optimization, stochastic optimization.

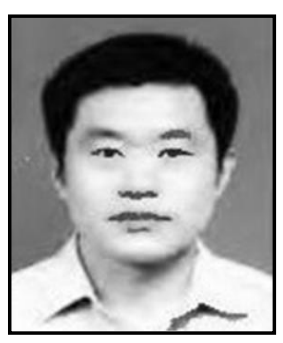

Sun Shufeng, works currently as a Distinguished Professor in College of Mechanical and Electrical Engineering, Wenzhou University. He has achieved the $\mathrm{PhD}$ degree in Mechatronic Engineering from Zhejiang University of Technology in 2013. His major interest work area is precision and ultra precision machining.

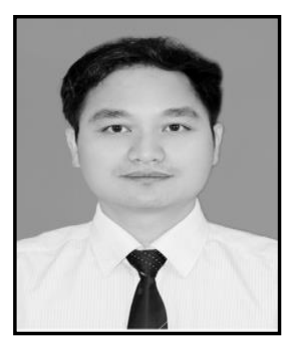

Jihong Pang is a researcher at Wenzhou University, China. $\mathrm{He}$ received the Ph.D. degree in mechanical engineering from Chongqing University of China. His major research interests include quality and reliability engineering, industrial engineering (IE), enterprise resource planning (ERP). 
International Journal of Grid Distribution Computing Vol. 8, No.6, (2015) 